\title{
Face recognition using PCA and different distance classifiers
}

\author{
Mohit P. Gawande*1, Prof. Dhiraj G. Agrawal*2 \\ *1 Shri sant Gadge baba College of Engineering, Bhusawal, India, \\ *2Shri sant Gadge baba College of Engineering, Bhusawal, India,
}

\begin{abstract}
A facial recognition system is a computer application for automatically identifying or verifying a person from a digital image or a video frame from a video source. One of the way is to do this is by comparing selected facial features from the image and a facial database. It is typically used in security systems and can be compared to other biometrics such as fingerprint or eye iris recognition systems. In this paper we focus on 3-D facial recognition system and biometric facial recognition system. We do critics on facial recognition system giving effectiveness and weaknesses. This paper also introduces scope of recognition system in India. Face recognition has received substantial attention from researchers in biometrics, pattern recognition field and computer vision communities.

Face recognition can be applied in Security measure at Air ports, Passport verification, Criminals list verification in police department, Visa processing, Verification of Electoral identification and Card Security measure at ATM's. Principal Component Analysis (PCA) is a technique among the most common feature extraction techniques used in Face Recognition. In this paper, a face recognition system for personal identification and verification using Principal Component Analysis with different distance classifiers is proposed. The test results in the ORL face database produces interesting results from the point of view of recognition success, rate, and robustness of the face recognition algorithm. Different classifiers were used to match the image of a person to a class (a subject) obtained from the training data. These classifiers are: the City-Block Distance Classifier, the Euclidian distance classifier, the Squared Euclidian Distance Classifier, and the Squared Chebyshev distance Classifier.
\end{abstract}

Keywords: Face Recognition, Eigen faces, Principal Component Analysis, Distance Measures.

\section{Introduction}

Person identification based on biometrics presents a challenging problem in the field of image analysis and computer vision, and as such has received a great deal of attention over the last few years because of its many applications in various domains. Biometrics is personal physical or biological measurements about an individual. Using biometrics for identifying human beings offers some unique advantages. A biometric based identification system has two modules. Enrollment module: A user's biometric data is acquired using a biometric reader and stored in a database. The stored template is labeled with a user identity (e.g., name, identification number etc. to facilitate authentication. Identification module: A user biometric data is once again acquired and the system uses this to either identify who the user is, or verify the claimed identity of the user. While identification involves comparing the acquired biometric information against templates corresponding to all users in the database, verification involves comparison with only those templates corresponding to the claimed identity. Thus, identification and verification are two distinct problems having their own inherent complexities. The unique features of biometric traits are extracted either from spatial domain or transform domain.

i)Spatial domain techniques are Principal Component Analysis, Independent Component Analysis, Linear Discriminative Analysis, and Singular Value Decomposition

ii) Transform domain techniques where in the biometric data from spatial domain is converted to transform domain viz., Fast Fourier Transform, Discrete Cosine Transform, Discrete Wavelet Transform, Dual Tree Complex Wavelet Transform etc. The features of the test image are compared with the data base images in matching section to identify a person using (i) Euclidean Distance, (ii) Hamming Distance, (iii) City Block Distance, (iv) Neural Networks etc.

\section{Face Recognition}

Face recognition is a form of biometric identification. A biometric is, "Automated methods of recognizing an individual based on their unique physical or behavioral characteristics." The process of facial recognition involves automated methods to determine identity, using facial features as essential elements of distinction. The automated methods of facial recognition, even though work very well, do not recognize subjects in the same manner as a human brain. The way we interact with other people is firmly based on our ability to recognize them. One of the main aspects of face identification is its robustness. Least obtrusive of all biometric 
measures, a face recognition system would allow a user to be identified by simply walking past a surveillance camera. robust face recognition scheme require both low dimensional feature representation for data compression purposes \& enhanced discrimination abilities for subsequent image retrieval. The representation methods usually start with a dimensionality reduction procedure since the high dimensionality of the original visual space makes the statistical estimation very difficult $\&$ time consuming [11, 13, and 27]. Face recognition is a study of how machines can recognize face, a task that humans perform naturally and effortlessly throughout lives[3]. A face recognition system identifies faces in images and videos automatically using computers. It consists of four parts: face detection, face alignment, facial feature extraction, and face classification. Face Detection: provides information about the location and scale of each detected face. In the case of video, the found faces may be tracked. In face alignment, facial components, such as eyes, nose, and mouth, and facial outline are located, and thereby the input face image is normalized in geometry and photometry. In feature extraction, features useful for distinguishing between different persons are extracted from the normalized face. In face classification, the extracted feature vector of the input face is matched against those of enrolled faces in the database, outputting the identity of the face when a match is found with a sufficient confidence or as an unknown face otherwise[10]. Depending on the application, a face recognition system can be working either on identification or verification mode. In a face identification application, the system recognizes an individual by matching the input image against images of all users in a database and finding the best match. In a face verification application the user claims an identity and the system accepts or rejects his (her) claim by matching the input image against the image that corresponds to this specific identity, which can be stored either in a database or an identification card (e.g. smart card). In other words, face identification is a one-to-many comparison that answers the question "Who is the person in the input image? Is he (she) someone in the database?", while face verification is a one-to-one comparison that answers the question "Is the person in the input image who he(she) claims to be?"[26]. A face recognition system usually consists of the following four modules[18]:

\section{Principle Component Analysis}

Feature extraction for face representation is one of the central issues to face recognition system. Among various solutions to the problem, the most successful seems to be those appearance-based approaches, which generally operate directly on images or appearances of face objects and process the image as two-dimensional patterns. The main trend in feature extraction has been representing the data in a lower dimensional space computed through a linear or non-linear transformation satisfying certain properties. Statistical techniques have been widely used for face recognition and in facial analysis to extract the abstract features of the face patterns. Principal Component Analysis is a main technique used for data reduction and feature extraction in the appearance-based approaches.

Eigenfaces built based on these technique, has been proved to be very successful [14]. Principal Component Analysis is one of the most valuable results from applied linear algebra. PCA is used abundantly in all forms of analysis - from neuroscience to computer graphics - because it is simple, non-parametric method of extracting relevant information from confusing data sets. With minimal additional effort PCA provides a roadmap for how to reduce a complex data set to a lower dimension to reveal the sometimes hidden, simplified structure that often underlie it [16]. It is one of the most successful techniques that have been used in image recognition and compression. PCA is a statistical method under the broad title of factor analysis. The purpose of PCA is to reduce the large dimensionality of the data space (observed variables) to the smaller intrinsic dimensionality of feature space (independent variables), which are needed to describe the data economically. This is the case when there is a strong correlation between observed variables. PCA is a statistical dimensionality-reduction method, which produces the optimal linear least-squares decomposition of a training set [9]. PCA is appropriate when we have obtained measures on a number of observed variables and wish to develop a smaller number ofunknown variables that will account for most of thevariance in the observed variables. PCA generates a set of orthogonal axes of projections known as the eigenvectors, of the input data distribution in the order of decreasing variances[18].

\section{Distance Classifiers}

Most face recognition methods from the last decade make decisions based on a distance measure. Images are projected down to a lower-dimensional feature space. Distances between feature space representations are used as the basis for recognition judgments. For example in an identification task, distance-based algorithms choose the gallery feature that is nearest to the probe feature. Distance measures are the last component of facial recognition. Images are projected into an eigenspace and represented as vectors. The distance between the vectors of two images is the similarity of the images. The dissimilarity distance between the image projection and known projections is calculated, the face image is then classified as one of the faces with minimum distance. On the other hand, classification is performed by comparing the projection vectors of the training face 
images with the projection vector of the input face image based on one of the distance classifiers between the faces classes and the input face image.

\subsection{Euclidean Distance Classifier}

Euclidean distance is the most common use of distance. When people talk about distance, this is what they are referring to. Euclidean distance, or simply 'distance', examines the root of square differences between the coordinates of a pair of objects. This is most generally known as the Pythagorean theorem. For testing we used the Euclidean distance classifier, for calculating the minimum distance between the test image and image to be recognized from the database. If the distance is small, we say the images are similar and we can decide which the most similar image in the database is. In simpler words, the Euclidean distance between the image projection and known projections is calculated; the face image is then classified as one of these faces with minimum Euclidean distance. Putting $\mathrm{p}=2$ in equation(10), we obtain the Euclidean distance, $\mathrm{d} 2$ as follows:

$d_{2}(x, y)=\sqrt{\sum_{i=1}^{l}\left|X_{i}-Y_{i}\right|^{2}}$

\subsection{The Squared Euclidean Distance Classifier}

Without the square roots, we obtain the Squared Euclidean distance Classifier as follows:

$d_{2}(x, y)=\sum_{i=1}^{l}\left|X_{i}-Y_{i}\right|^{2}$

\subsection{City-Block Distance Classifier}

City-Block Distance Classifier, Manhattan Distance Classifier, also called, rectilinear distance, L1 distance, L1 norm, Manhattan length. It represents the distance between points in a city road grid. It examines the absolute differences between the coordinates of a pair of objects as follows:

$d_{1}(x, y)=\sum_{i=1}^{l}\left|X_{i}-Y_{i}\right|$

\subsection{Chebyshev Distance Classifier,}

maximum value Distance In mathematics, Chebyshev distance, Maximum metric, or L $\infty$ metric is a metric defined on a vector space where the distance between two vectors is the greatest of their differences along any coordinate dimension.

The Chebyshev distance between two vectors or points $\mathrm{x}$ and $\mathrm{y}$, with standard coordinates $\mathrm{xi}$ and $\mathrm{yi}$, respectively, is:

$\lim _{K \rightarrow \infty}\left(\sum_{i=1}^{K}\left|X_{i}-Y_{i}\right|^{K}\right)^{1 / K}$

Hence it is also known as the L $\infty$ metric. Taking the squares of equation (14), we obtain the squared

Chebyshev Distance Classifier. Chebyshev distance is also called the Maximum value distance, defined on a vector space where the distance between two vectors is the greatest of their differences along any coordinate dimension. In other words, it examines the absolute magnitude of the differences between the coordinates of a pair of objects. the same image

\section{Results And Discussion}

Figure (2) shows a Schematic diagram of a face recognizer. This illustrated as follows: In the recognition phase (or, testing phase), given a test image of a known person. As in the training phase, we should compute the feature vector of this person using PCA, then compute the

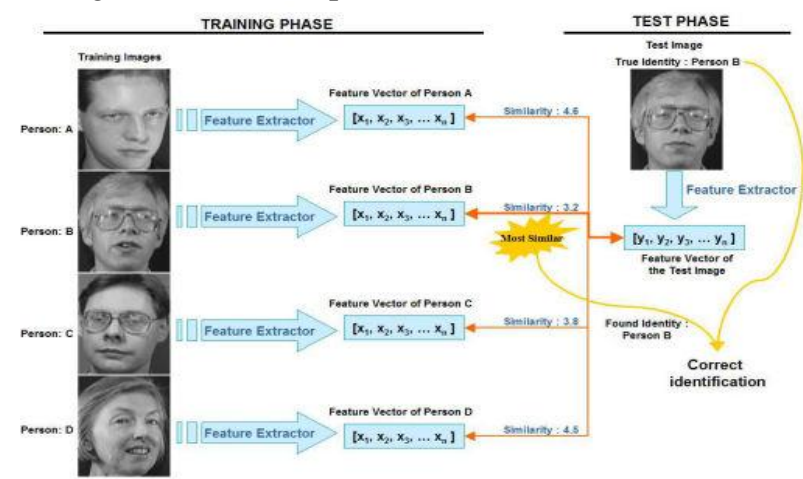

Fig. 2 Schematic diagram of a face recognizer

similarities between the feature vector of a test image and all the feature vectors in the raining set. The similarities between feature vectors were computed using the 4 distance classifiers discussed in the previous 
section. On the other hand, recognition is performed by projecting a new image into the subspace spanned by the eigenfaces and then classifying the face by comparing its position in the face space with the positions of the known individuals.
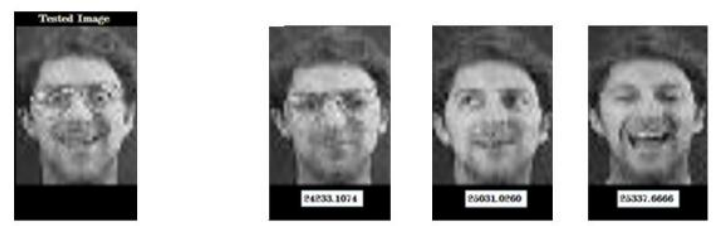

(A) City- Block Distance Classifier.
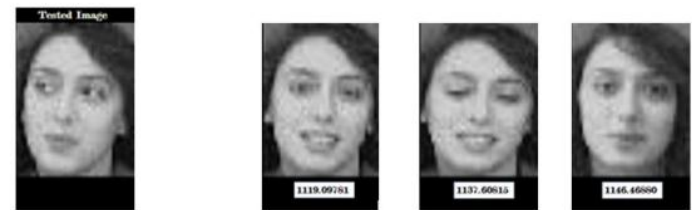

(B) Euclidian Distance Classifier
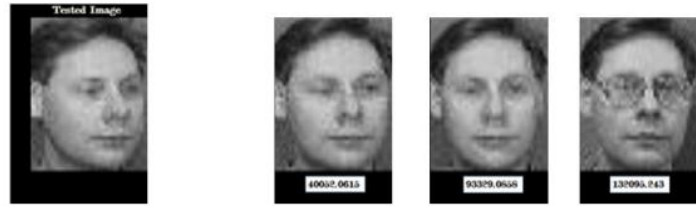

(C): Chebyshev Distance Classifier (squared).

Figure (3): Screen Shots displays full recognitions with the nearest three projected distances. The three images in the right identified from the trained

Database
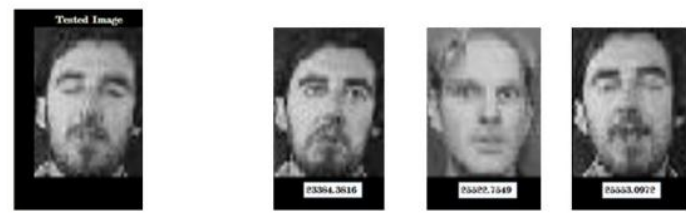

(A): City-Block Distance Classifier.
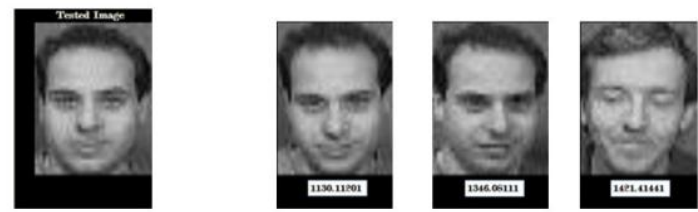

(B): Euclidian Distance Classifier.
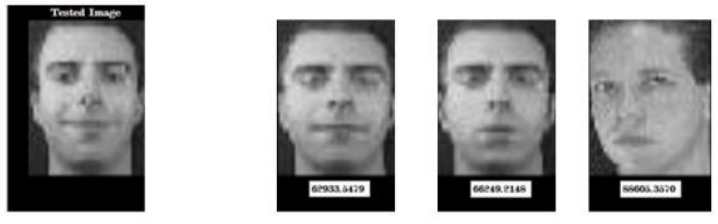

(C): Chebyshev Distance Classifier (squared).

Figure (4): Screen Shots displays an error recognition in some samples. The three images in the right identified from the trained Database.

\section{Conclusions}

Face recognition can be applied in Security measure at Air Ports, Passport verification, Criminals list verify police department, Visa Processing, Verification of Electoral identification and Card Security measure at ATM's. Face recognition has received substantial attention from researches in biometrics, pattern recognition field and computer vision communities. In this paper, face recognition using principal component analysis was implemented using 4 distance classifiers on the ORL database were used to see the performance of the Principal Component Analysis based face recognition system. A system the distance measures for each image will perform better than a system that only uses one. The experiment show that PCA gave better results with Euclidian distance classifier and the squared Euclidian distance classifier than the City Block distance classifier, which gives better results than the squared Chebyshev distance classifier. On the other hand, the recognition rate 
using the Euclidian and the Squared Euclidian distance classifier are the same and is higher than the recognition rate using City-Block distance classifier which is higher than its counterpart using the squared Chebyshev distance classifier.

\section{References}

[1] M. Abul Kashem, M. Akhter, S. Ahmed, and M. Alam, "Face Recognition System Based on Principal Component Analysis (PCA) with Back Propagation Neural Networks(BPNN)", Canadian Journal on Image Processing and Computer Vision Vol. 2, No. 2, No. 4, 2011.

[2] J. Meng, and W. Zhang, "Volume measure in 2DPCA-based face recognition", Pattern Recognition Letters 28 (2007) 1203-1208.

[3] I. Timotius, I. Setyawan, and A. Febrianto, "Face Recognition between Two Person using Kernel Principal Component Analysis and Support Vector Machines", International Journal on Electrical Engineering and Informatics - Volume 2, 2010.

[4] T. Mandal and Q. Jonathan Wu, "Face Recognition using Curvelet Based PCA", IEEE, 2008.

[5] O. Gervei, A. Ayatollahi, and N. Gervei, “\#D Face Recognition Using Modified PCA Methods", World Academy of Science, Engineering and Technology 632010.

[6] N. Nain, P. Gour, N. Agarwal, R. Talawar, and S. Chandra, "Face Recognition using PCA and LDA with Singular Value Decomposition (SVD) using 2DLDA", Proceedings of the World Congress on Engineering 2008 Vol1, London. U.K.

[7] M. Sharkas, "Applicaion of DCT Blocks with Principal Component Analysis for Face Recognition", Proceedings of the 5th WSEAS int. Conf. on Signal, Speech and Image Processing, Corfu, Greece, (pp107-111), 2005.

[8] A. Eleyan, and H. Demirel, "PCA and LDA Based Face Recognition Using Feedforward Neural Network Classifier", MRCS 2006, LNCS 4105, pp. 199-206, 2006. c Springer- Verlag Berlin Heidelberg 2006.

[9] H. Moon, and P. Phillips, "Computational and performance aspects of PCA-based face-recognition algorithms", Perception, 2001, volume 30, pp303-321.

[10] A. MANE, R. MANZA, and K. KALE, "Human Face Recognition Using Superior Principal Component Analysis (SPCA)", International Journal of Computer Theory and Engineering, Vol. 2, No. 5, 2010.

[11] K. Karande, and S. Talbar, "'Face Recognition under Variation of Pose and Illumination using Independent Component Analysis", ICGST - GVIP, ISSN 1687-398X, Volume (8), Issue (IV), 2008.

[12] J. Mazanec, and M. Melisek, "Support Vector Machines, PCA and LDA in Face Recognition". Journal of ELECTRICAL ENGINEERING, pp. 203-209 VOL. 59, NO. 4, 2008.

[13] J. Ghorpade, and S. Agarwal, "SOM and PPCA Approach for Face Recognition - A Survey". International Journal of Computer Trends and Technology - March to April Issue - 2011

[14] Y. Ghassabeh, H. Moghaddam, and M. Teshnehlab, “Adaptive Modified PCA for Face Recognition”. MVA2007 IAPR Conference on Machine Vision Applications, May 16-18, 2007, Tokyo, JAPAN.

[15] C. Han, "Modular PCA Face Recognition Based on Weighted Average". Modern Applied Science, Vol. 3, No. 11, November 2009.

[16] D. Chen, and H. Jiu-qiang, "An FPGA-based face recognition using combined 5/3 DWT with PCA methods". Journal of Communication and Computer, ISSN 1548-7709, USA, Volume 6, No. 10 (Serial No. 59), Oct. 2009.

[17] Y. Wen, and P. Shi, "Image PCA: A New Approach for Face Recognition" c IEEE, 2007.

[18] T. Deb, D. Bhattacherjee, M. Nasipuri, D. Basu, and M. Kundu, "An enhanced face recognition technique based on Overlapped Modular PCA approach for cropped Log-polar images". International Journal of Recent Trends in Engineering, Vol 2, No. 1, November 2009.

[19] M. Turk, and A. Pentland, "Eigenfaces for Recognition". Journal of Cognitive Neuroscience Volume 3, Number 1 c1991 Massachusetts Institute of Technology.

[20] J. Gan, D. Zhou, and C. Li, "A Method For Improved PCA in Face Recognition”. International Journal of Information Technology Vol. 112005 . 